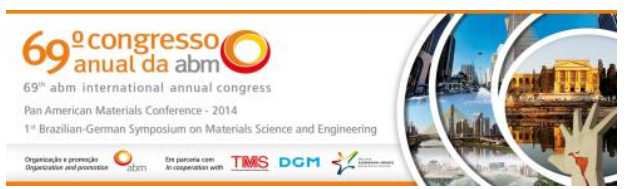

Tema: Gestão Sistêmica

\title{
TECNOLOGIA DE ARMAZENAGEM APLICADA NO SUPORTE A GESTÃO DE FERRAMENTAS DE USINAGEM NO SETOR AUTOMOTIVO*
}

\section{Resumo}

Alexandre Rodizio Bento ${ }^{1}$ Sérgio Luís Tambosi ${ }^{2}$ Élcio Miguel Prus ${ }^{3}$

A competitividade do setor automotivo exige maior agilidade na gestão de ferramentas de usinagem. Para atender esta necessidade flexibilizar e automatizar o processo de gestão de armazenagem é fator primordial. As ferramentas de usinagem muitas vezes têm custo elevado e a necessidade de controle constante é imprescindível no setor automotivo. A padronização de armazenagem aliada a um equipamento específico para controlar ferramentas surge como uma opção. Pois, a troca de produtos deve ser ágil durante a fabricação para evitar perdas e máquinas ociosas no chão de fábrica. Assim, suprir esta necessidade com velocidade é essencial e de suma importância para a integração entre os departamentos de produção e logística. Neste cenário, a tecnologia de armazenagem permite agilizar e flexibilizar a gestão de ferramentas de usinagem, além de possibilitar ganhos de produção. Neste trabalho, apresenta-se a substituição do processo manual de armazenagem de ferramentas de usinagem baseado em caixas e prateleiras, por um sistema com tecnologia de armazenagem vertical. Esta substituição permite automatizar o controle de ferramentas, reduzir o custo de estoque e flexibilizar a troca de produtos na produção. Os resultados deste processo permitem garantir a melhor gestão das ferramentas de usinagem, evitar paradas nas linhas, reduzir custos operacionais e manter estoques em perfeito equilíbrio, entre o aumento de produtividade e a redução de custos.

Palavras-chave: Tecnologia de armazenagem; Ferramentas de usinagem; Setor automotivo.

\section{STORAGE TECHNOLOGY APPLIED IN MANAGEMENT TOOLS SUPPORT IN MACHINING AUTOMOTIVE}

\begin{abstract}
The competitiveness of the automotive industry requires greater flexibility in the management of machining tools. To meet this need flexibility and automate the management of storage is a key factor. Because the machining tools often have high costs the need for constant control is essential in the automotive sector. The standardization of storage allied coupled to a specific equipment to control tools appears as an option. Because the exchange of products must be agile during manufacturing to avoid losses and idle machines on the factory floor. So to fulfill this need with speed it is essential the integration between departments of production and logistics. In this scenario, the storage technology allows faster and more flexible management of machining tools, as well as, enable production gains. In this paper, we present the replacement of manual storage process of machining tools based on boxes and shelves, for a system with vertical storage technology. This substitution allows the automation control tools, and further reduce the inventory cost and easing the exchange of products in production. The results of this use permit to ensure better management of machining tools avoiding stops in lines, reducing operating costs and allowing keeping inventories in perfect balance between increasing productivity and reducing costs.

Keywords: Technology of storage, machine tools, automotive.

1 Processamento de Dados, Mestre em Desenvolvimento de Tecnologia, Faculdades Santa Cruz, Curitiba, PR, Brasil.

2 Processamento de Dados, Mestre em Informática Aplicada, Faculdades Santa Cruz, Curitiba, PR, Brasil.

3 Bacharel Sistemas de Informação, Mestre em Eng.de Produção, Faculdades Santa Cruz, Curitiba, PR, Brasil.
\end{abstract}

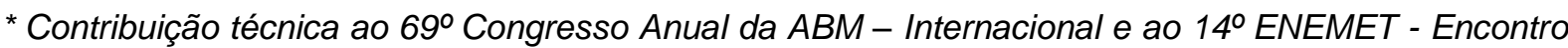
Nacional de Estudantes de Engenharia Metalúrgica, de Materiais e de Minas, 21 a 25 de julho de 2014, São Paulo, SP, Brasil.
} 


\section{INTRODUÇÃO}

O crescimento do mercado automotivo mundial faz ampliar a competitividade deste setor, que busca por ferramentas tecnológicas e estratégias de negócios para atender as demandas de produção do mercado em expansão. Esta busca gera aumento de produtividade e deve estar alinhada diretamente aos níveis de estoque, pois a falta de um determinado recurso (matéria-prima, máquinas, ferramentas, pessoas e outros) pode comprometer a produção. Além da matéria prima as ferramentas utilizadas nas máquinas (centro de usinagem) para produzir peças são de suma importância [1]. Neste contexto, a tecnologia de armazenagem de ferramentas de usinagem surge como um dos requisitos primordiais para atender as necessidades de produção no prazo estipulado e com maior agilidade.

As ferramentas de usinagem são constituídas de arestas cortantes, destinada a remoção do cavaco muito utilizada no setor automotivo e importante no processo de manufatura [2]. Uma ferramenta de baixa qualidade ou vida útil menor ao padrão estipulado pelo fornecedor da mesma, pode acarretar em perda ou retrabalho com custo considerável para o setor. Devido a isto, a necessidade de controlar as ferramentas de usinagem se torna cada vez imprescindível para suprir as demandas dos clientes e reduzir os custos de produção. Outro fator que pode onerar é o prazo de fabricação da ferramenta aliado ao alto custo.

Para controlar as ferramentas de usinagem de forma eficiente, a padronização na armazenagem surge como uma opção [3]. Alta rotatividade indica que armazenagem deve ser específica para cada ferramenta e de fácil acesso, pois a mudança de uma peça no centro de usinagem tem que ser ágil, evitando assim paradas ou gargalos na linha de produção, bem como a ociosidade do centro de usinagem.

Uma linha de produção parada pode ter diversas ocorrências, sendo as mais comuns a falta de matéria prima ou ferramenta e problemas com manutenção [4].

A ocorrência do evento de parada deve ser sinalizada pela produção para os responsáveis dos departamentos de logística, compras e manutenção, conforme a ocorrência evidenciada. A integração entre esses departamentos pode contribuir para agilizar a entrega da ferramenta ou peça de reposição junto ao fabricante. Manter estoque das ferramentas que são utilizadas com mais frequência é uma opção que pode evitar a parada da linha.

O primeiro objetivo deste trabalho é apresentar o processo manual de armazenagem de ferramentas de usinagem baseado em prateleiras. Após esta apresentação substituir o processo manual por um sistema automatizado vertical. Com isso, pretende-se demonstrar maior agilidade na gestão das ferramentas como a entrada e saída, reduzir o custo de estoques e tempo de operação.

\subsection{TECNOLOGIA DE ARMAZENAGEM DE FERRAMENTAS}

A gestão das ferramentas de usinagem é primordial para a produção de peças e componentes automotivos, devido aos centros de usinagem utilizados no setor automotivo, comportarem até 50 ferramentas no magazine para executar as operações de usinagem. Esta grande utilização de ferramentas torna o controle das mesmas imprescindíveis para a continuidade das atividades produtivas no setor.

Para Machado [5] os centros de usinagem são equipamentos com alto custo de investimento que eliminam as operações manuais tais como: furar, fresar e rebaixar a peça. Estas operações retiram o cavaco da peça e fornece o estado de acabada, agregando alto valor ao produto final [6]. Alguns centros de usinagem podem ser

\footnotetext{
* Contribuição técnica ao $69^{\circ}$ Congresso Anual da ABM - Internacional e ao 14ํㅡㄹ ENEMET - Encontro Nacional de Estudantes de Engenharia Metalúrgica, de Materiais e de Minas, 21 a 25 de julho de 2014, São Paulo, SP, Brasil.
} 


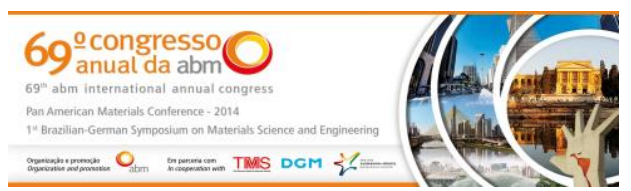

equipados com magazine de ferramentas multifuncionais, também conhecido como trocador automático de ferramenta (TAF). Por isso a necessidade de controlar armazenagem das ferramentas torna-se um desafio diário.

A armazenagem de ferramentas de forma correta pode permitir maiores lucros a indústria automotiva. Segundo Pozo [7] armazenagem pode propiciar sucesso a empresa e conquistar novos clientes, pois elimina a possibilidade de parar a linha de produção por falta de ferramenta. Esta disposição de ferramentas pode evitar problemas futuros entre o fornecedor e cliente. Arbache [8], a armazenagem pode assegurar o controle de produtos desde a recepção (entrada) até distribuição (saída). Além disso, dispõem de quatro funções básicas: receber, estocar, administrar pedidos e expedir produtos.

A tecnologia inserida no controle de armazenagem de produtos proporciona maior controle e ainda auxilia no planejamento e na tomada de decisão estratégica [9]. Para Banzato [10] armazenagem exige muito mais que procedimentos automatizados e deve inserir ferramentas tecnológicas que permitem tomar decisões ágeis e inteligentes. Estas ferramentas podem interferir na rentabilidade da indústria, pois pode afetar o processo de produção e onerar o custo do produto final.

Já para Dias [11] armazenagem pode definir o compromisso da indústria entre os custos agregados nas operações de produção ou manter altos níveis de estoque. Para escolher a melhor opção é necessário ter o controle de todas as variáveis que compõem os custos de armazenagem e as demandas de produção.

As atividades principais que compõem armazenagem são receber (identificar e inspecionar o material), estocar (classificar o estoque de matéria prima ou ferramentas), distribuir (expedir a peça acabada) [12]. Quando estas atividades são executadas de forma assertiva podem trazer os seguintes benefícios: Aproveitar melhor o espaço, reduzir custo com movimentação de materiais, facilitar auditoria do processo e minimizar as perdas.

O processo de movimentação dos produtos dentro da indústria pode ser executado de três formas: manual (interferência humana), mecanizada (equipamentos operados por pessoas) e automatizados (por meio de ferramentas tecnológicas) [13]. Estas formas de movimentação são utilizadas para guardar, localizar, manusear, distribuir os produtos com objetivo de atender as necessidades operacionais tais como: entrada por nota fiscal, demanda da produção e venda de produtos.

A tecnologia está cada vez mais embutida nos processos de armazenagem com equipamentos informatizados que fazem de forma automática a gestão das ferramentas de usinagem em tempo real [14]. A maioria das ferramentas tem tamanho pequeno, mas necessitam armazenagem em grande quantidade devido a demanda da produção. A tecnologia aliada armazenagem pode ser um diferencial na gestão das ferramentas, além de pouca interferência humana, ocupa espaço reduzido, controla entrada e saída de várias ferramentas de forma ágil.

Desta forma, utilizar equipamentos de armazenagem sistematizados pode ser a decisão certa, devido aos múltiplos benefícios que a tecnologia proporciona. Antes da escolha final, devem ser avaliados todos os pros e contra deste investimento em automação, pois os benefícios de controle e agilidade podem não ser os mesmos para todas as indústrias existentes no mercado.

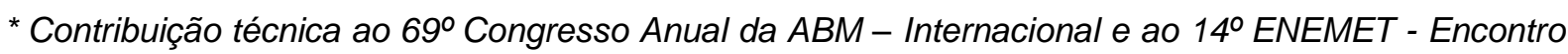
Nacional de Estudantes de Engenharia Metalúrgica, de Materiais e de Minas, 21 a 25 de julho de 2014, São Paulo, SP, Brasil.
} 


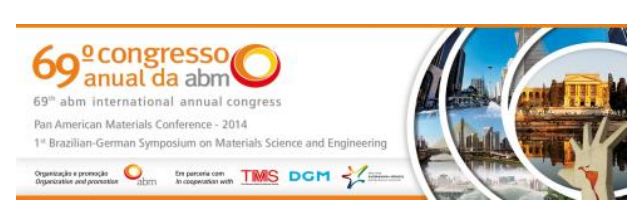

\section{MATERIAIS E MÉTODOS}

Para apresentar a tecnologia de armazenagem de ferramentas de usinagem foi escolhida uma indústria do setor automotivo localizada na região sul do Brasil. As ferramentas são utilizadas em grande quantidade nos centros de usinagem da indústria escolhida. A indústria tem em seu parque fabril 620 centros de usinagem que utiliza 25.000 ferramentas na produção de 1.164 .000 peças e componentes automotivos por mês. Devido à grande demanda dos clientes as linhas de produção não podem parar por falta de matéria prima, ferramentas ou outras situações.

A gestão de armazenagem das ferramentas de usinagem surge como situação primordial para continuidade da produção. A indústria selecionada tem no estoque a quantidade de 26.648 ferramentas, sendo 6.729 modelos diferentes resultando no valor total de $R \$ 3.000 .000 .00$ em estoque. O alto valor de cada ferramenta de usinagem impõem a gestão controlada com estoques referentes a dois dias de produção em casos de ferramentas com maior tempo de fabricação onde o próprio fornecedor armazena este estoque. Estes casos são tratados por meio de contratos feitos entre o departamento de compras da indústria e o fornecedor.

$O$ processo de armazenagem tradicional das ferramentas de usinagem, das operações de receber até armazenar a ferramenta, integrado com o processo de solicitar a disponibilizar a ferramenta para uso na linha de produção é demonstrado na figura 1.
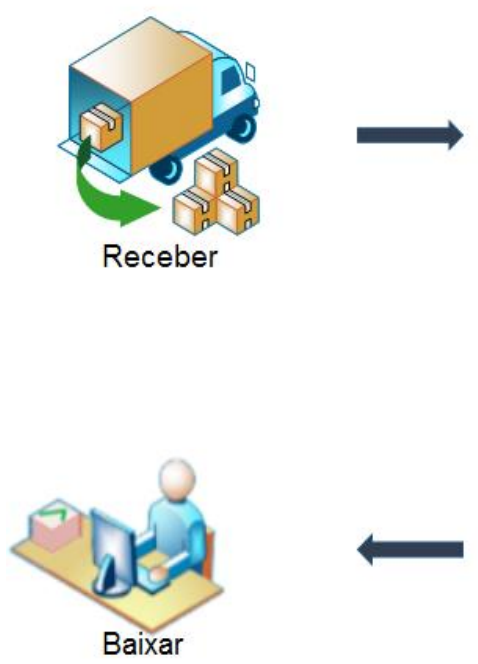
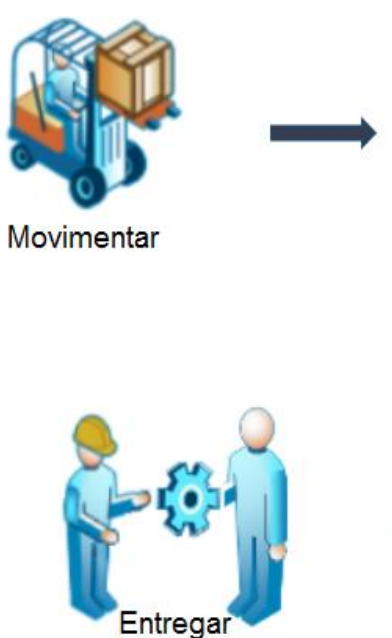

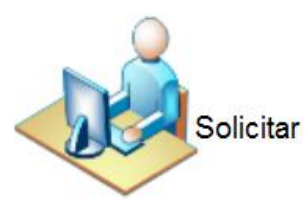

I
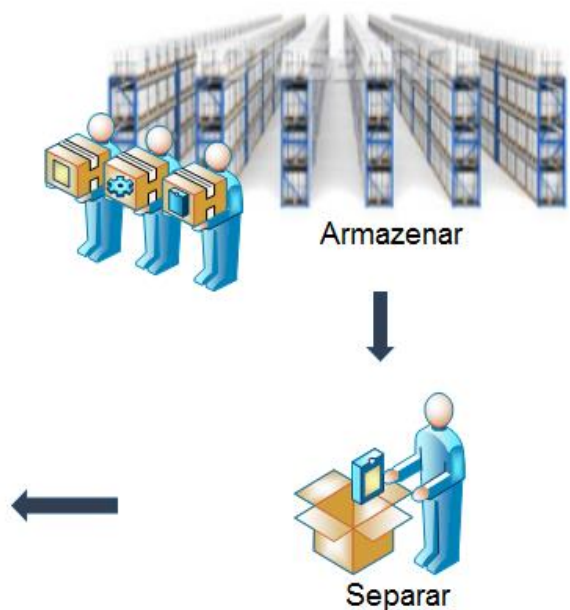

Figura 1. Processo armazenagem tradicional.

A figura 1 demonstra o processo de armazenagem tradicional do recebimento da ferramenta até a baixa da mesma no sistema. O primeiro processo é receber a ferramenta e movimentar com auxílio de uma empilhadeira (máquina utilizada para carregar, movimentar e descarregar mercadorias) até ao almoxarifado para armazenar as ferramentas em prateleiras e caixas. A armazenagem em prateleira ocupa uma área de $9 \times 3$, ou seja, 27 metros quadrados e dispõe de três funcionários para fazer a gestão de todas as ferramentas. A solicitação de

\footnotetext{
* Contribuição técnica ao $69^{\circ}$ Congresso Anual da ABM - Internacional e ao 14ํㅡㄹ ENEMET - Encontro Nacional de Estudantes de Engenharia Metalúrgica, de Materiais e de Minas, 21 a 25 de julho de 2014, São Paulo, SP, Brasil.
} 


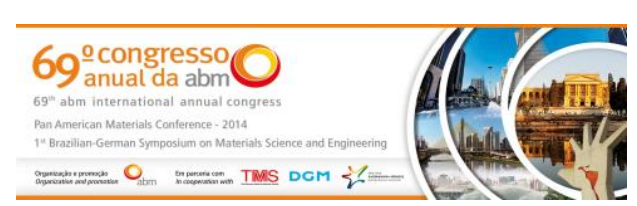

ferramentas pode ser efetuada por qualquer funcionário com acesso ao sistema de estoque. Esta solicitação gera uma pendência via sistema para o almoxarifado que procura efetuar a separação da ferramenta solicitada para posterior entrega ao solicitante. Após a entrega é efetuada a baixa da quantidade no sistema e de forma automática atualiza o saldo em estoque e contabiliza a data, código e para qual linha de produção foi utilizada a ferramenta, todos estes dados são informados na inclusão da solicitação. O tempo médio de todos os processos citados é de 10 minutos. O grande volume de mercadorias armazenadas em conjunto com as ferramentas no mesmo almoxarifado pode contribuir para atrasar a separação e entrega das ferramentas.

O fluxo para armazenar ferramentas com controle sistematizado vertical, permite automatizar os processos logísticos e ainda reduzir custos e agilizar todas as etapas para solicitar ferramentas e entregar a mesma em menor tempo.

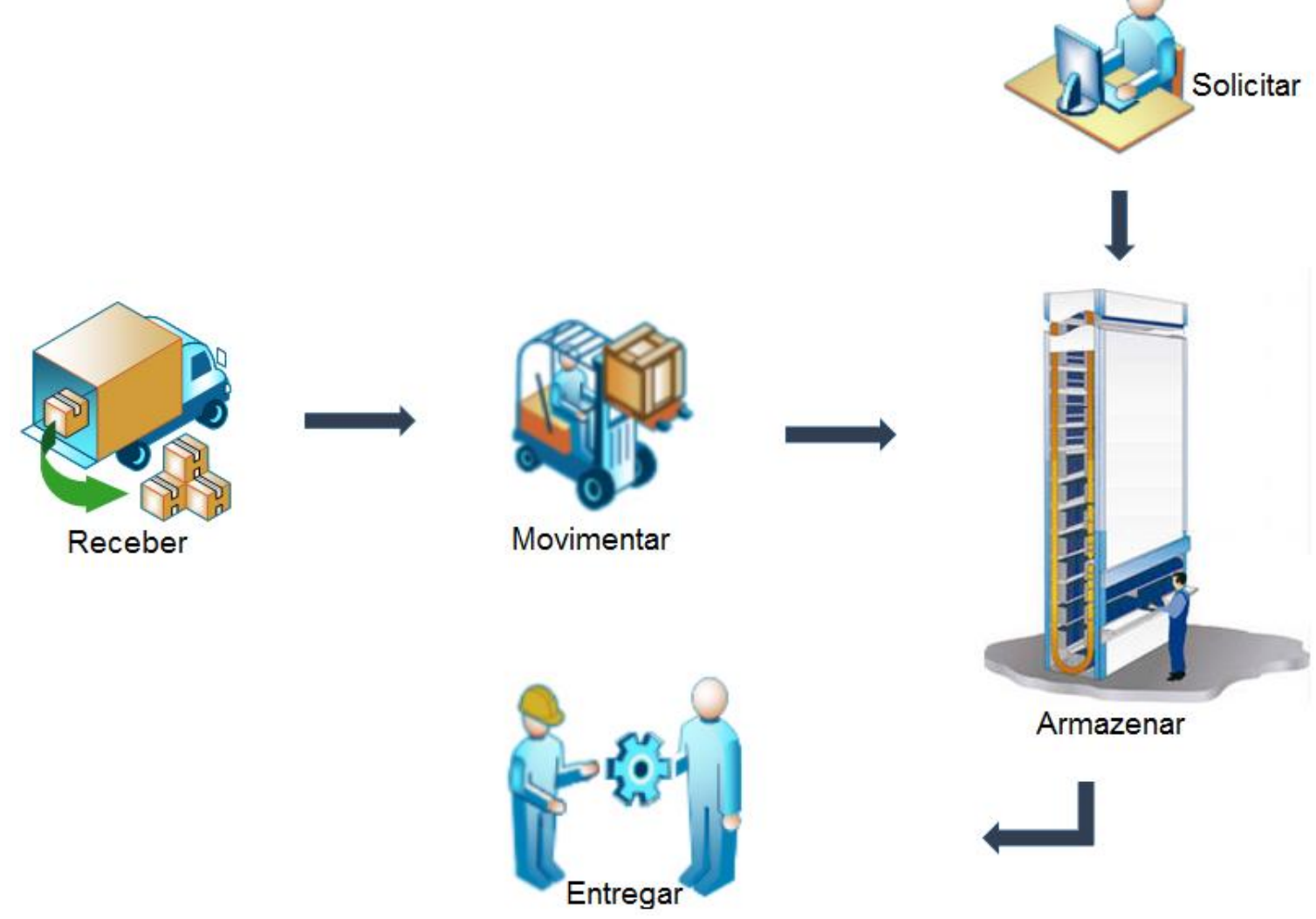

Figura 2. Processo de armazenagem sistematizado.

A figura 2 demonstra o processo de armazenagem sistematizado do recebimento da ferramenta até entrega da mesma ao solicitante. Para este processo foi criado um novo departamento com o nome almoxarifado de ferramentas em uma nova localização exclusiva para a gestão de armazenagem das ferramentas. Este almoxarifado está localizado ao lado da central de ferramentas, local responsável por efetuar a manutenção e troca das ferramentas com avaria ou desgaste por novas. Assim, somente a central de ferramentas pode solicitar novas ferramentas ao almoxarifado.

Ao receber as ferramentas e movimentar com empilhadeira até o almoxarifado de ferramenta, apenas um funcionário efetua armazenagem e o controle do equipamento vertical de forma sistematizada. O equipamento tem 67 bandejas flexíveis que podem alterar de tamanho, conforme a espessura ou quantidades de

\footnotetext{
* Contribuição técnica ao $69^{\circ}$ Congresso Anual da ABM - Internacional e ao 14ํㅡㄹ ENEMET - Encontro Nacional de Estudantes de Engenharia Metalúrgica, de Materiais e de Minas, 21 a 25 de julho de 2014, São Paulo, SP, Brasil.
} 


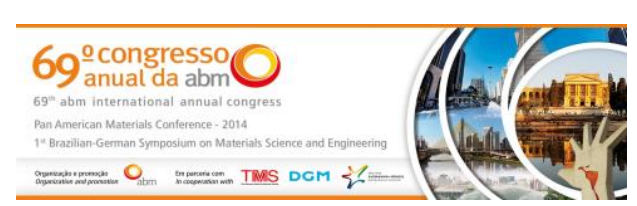

ferramentas a serem armazenadas. Além disso, o armazém vertical tem um painel com toque na tela que permite controlar todas as bandejas e monitorar os níveis de estoque. Com isso, é possível fazer a gestão das ferramentas de usinagem de forma rápida. O espaço reduzido é outra vantagem do armazém vertical que ocupa uma área de 3 metros de largura por 3,1 metros de profundidade por 8 metros de altura, ou seja, apenas 9,3 metros de área ocupada no chão. Devido o armazém vertical ser automatizado facilita o controle e armazenagem das ferramentas por bandeja.

A solicitação de ferramentas é efetuada pela central de ferramentas no sistema, que está interligado com o armazém vertical, e disponibiliza a ferramenta solicitada de forma automática. $O$ funcionário retira a ferramenta do armazém vertical e entrega a mesma na sala ao lado. O controle de baixa da movimentação e contabilização no sistema das ferramentas é feito a partir do armazém vertical. Todos os processos desde a solicitação até a entrega da ferramenta duram apenas 2 minutos.

\section{RESULTADOS E DISCUSSÕES}

Para uma melhor comparação entre o processo manual de armazenagem e o sistematizado proposto neste trabalho é necessário apresentar os fluxogramas destes processos. Ambos são representados na figura 3 onde pode-se observar que a principal contribuição está no armazém vertical, que permite minimizar os custos operacionais, agilizar o processo para solicitar ferramenta, evitar paradas na linha de produção por falta de ferramenta, reduzir os níveis e custos dos estoques.

A figura 3 faz uma comparação entre o fluxo de armazenagem tradicional (a) e sistematizado (b). Pode-se observar algumas contribuições com a utilização da tecnologia do armazém vertical no processo de armazenagem de ferramentas de usinagem. A primeira contribuição é o uso do armazém vertical em substituição as prateleiras e caixas do almoxarifado que permite reduzir custos operacionais de dois funcionários. O custo de cada funcionário é de $R \$ 2.000,00$ (dois mil reais) sendo a economia total de $\mathrm{R} \$ 4.000,00$ (quatro mil reais) por mês. Além disso, o espaço físico, reduz de forma significativa, se comparada com o armazém tradicional que atinge 27 metros de área ocupada, já no sistematizado apenas 9,3 metros por 8 metros de altura, um ganho de espaço considerável já que a cobertura da indústria tem 9 metros de altura.

A agilidade durante todo o processo de armazenagem do recebimento a baixa da ferramenta são proporcionados pela tecnologia do armazém vertical, pois se comparado o tempo no processo tradicional (a) atinge 10 minutos, enquanto no sistematizado (b) apenas 2 minutos um ganho de $80 \%$ no processo. Este ganho pode se evidenciar devido ao armazém sistematizado já estar integrado ao sistema de gestão da indústria o que permite separar e efetuar a baixa da ferramenta de forma automática, sem interferência humana nestes processos. Outro quesito importante foi a criação do almoxarifado exclusivo para ferramentas, devido a importância das mesmas para a produção. A nova localização do almoxarifado também contribuiu para reduzir o tempo na retirada da ferramenta.

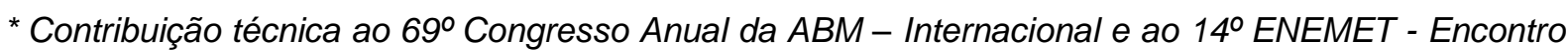
Nacional de Estudantes de Engenharia Metalúrgica, de Materiais e de Minas, 21 a 25 de julho de 2014, São Paulo, SP, Brasil.
} 


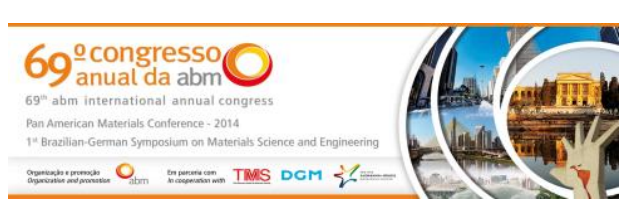

possíveis paradas da linha de produção por falta de ferramentas. Não se pode esquecer que o armazém vertical está integrado ao sistema de gestão que permite maior controle nos relatórios de estoque tais como: entrada, saída e média de utilização das ferramentas de usinagem.

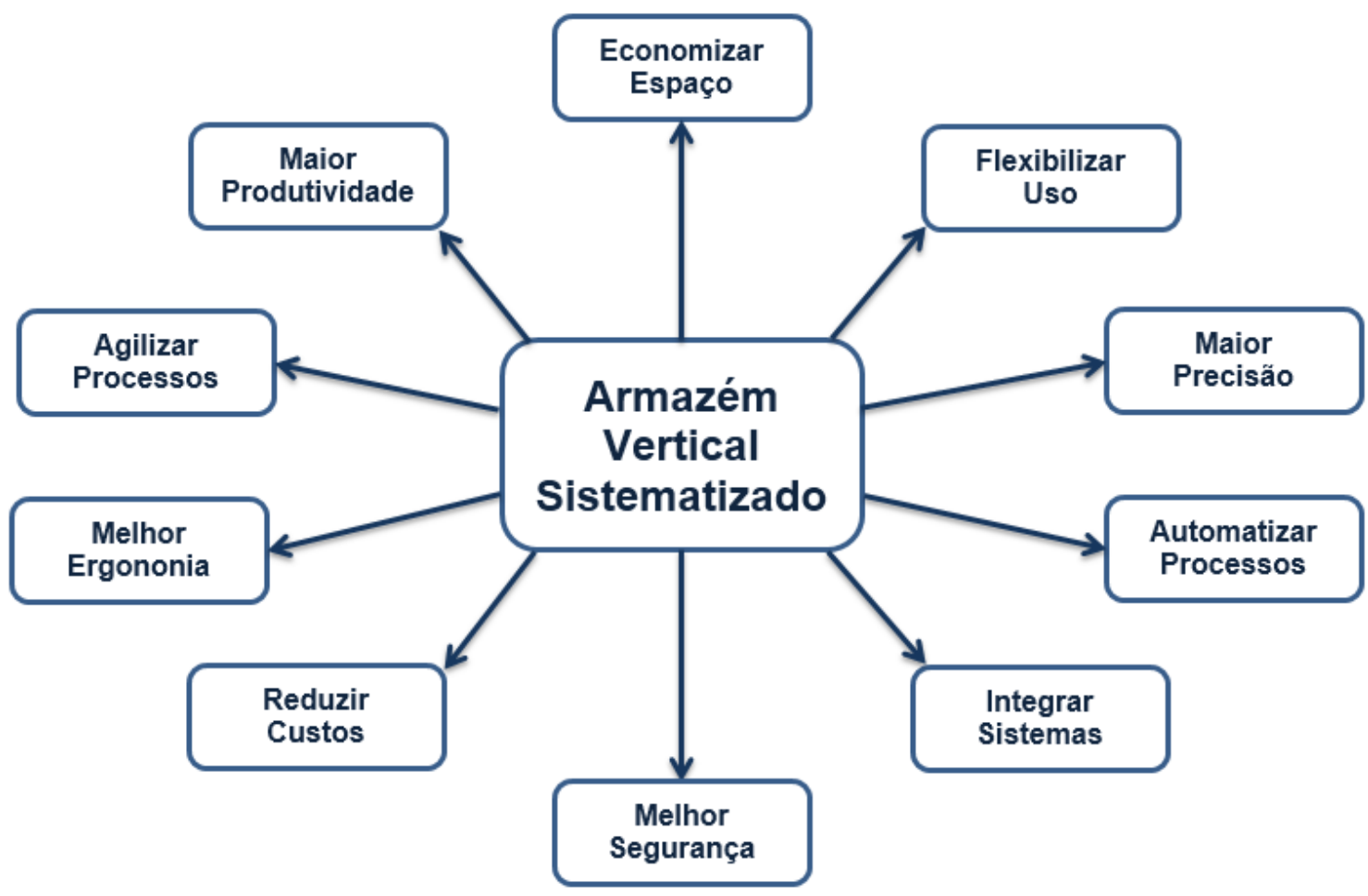

Figura 4. Benefícios do armazém vertical sistematizado.

A figura 4 apresenta os benefícios da utilização do armazém vertical sistematizado no suporte a gestão de ferramentas de usinagem no setor automotivo. O armazém sistematizado permite economizar espaço por ser vertical e utilizar várias gavetas rolantes internamente para flexibilizar a utilização de ferramentas de vários tamanhos com maior precisão. Os processos são automáticos e permite integrar aos sistemas de gestão com maior segurança e estes aspectos contribuem para reduzir custos. A questão ergonômica no trabalho é essencial para evitar doenças e agilizar os processos de armazenagem com maior produtividade de peças e componentes automotivos.

Assim, todos os aspectos citados e associados demonstram ganhos de forma orquestradas nos processos de armazenagem de ferramentas de usinagem de forma integrada ao sistema da indústria. A tecnologia de armazenagem sistematizada apresenta-se superior sob diversos aspectos analisados, que permitiu sua aplicação de forma imediata.

\section{CONCLUSÃO}

O setor automotivo busca controles de armazenagem sistematizados para agilizar os processos internos e a gestão dos estoques das ferramentas de usinagem com objetivo de reduzir custos e diminuir o tempo de execução nos processos de armazenagem. Este é o caso da tecnologia embutida ao armazém vertical, que se bem aplicada e utilizada por ser um diferencial estratégico para o setor.

* Contribuição técnica ao 69 Congresso Anual da ABM - Internacional e ao 14ํㅡㄹ ENEMET - Encontro Nacional de Estudantes de Engenharia Metalúrgica, de Materiais e de Minas, 21 a 25 de julho de 2014, São Paulo, SP, Brasil. 


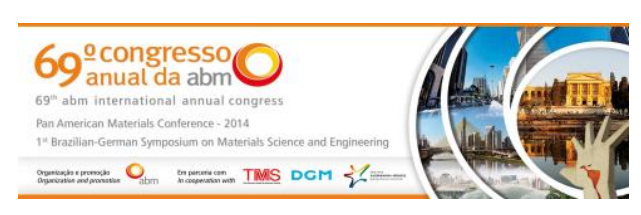

Os processos sistematizados de armazenagem de ferramentas estão com ciclos de vida menores, onde a ferramenta se torna a matéria prima principal. Para atender esta demanda a gestão de armazenagem das ferramentas é um desafio para as indústrias automotivas que desejam permanecer no mercado onde a competitividade é acirrada entre os concorrentes.

O armazém vertical sistematizado apresentou ganhos como redução de tempo em $80 \%$, custo operacionais $66 \%$ e espaço ocupado. Além dos benefícios já descritos proporciona maior agilidade, flexibilidade e facilidade de uso com tecnológica moderna agregada aos métodos de armazenagem de ferramentas de usinagem.

Assim, utilizar armazém vertical sistematizados no setor automotivo é um quesito para garantir maiores lucros. Além de ser um diferencial perante os concorrentes do setor. A aplicação desta ferramenta tecnológica nos processos de armazenagem garante melhorias na gestão de estoques e consequentemente maior segurança e confiabilidade para a indústria.

\section{REFERÊNCIAS}

1 Magalhães JSF. Sistema Variante para Seleção de Maquinas -Ferramenta. 2008. 72f. Dissertação (Mestrado em Engenharia Mecânica) - Programa de PósGraduação em Engenharia Mecânica da Universidade Federal do Paraná, Curitiba, 2008.

2 Rodrigues AR. Estudo da Geometria de Arestas de Corte Aplicadas em Usinagem com Alta Velocidade de Corte. 2005. 227f. Tese (Doutorado em Engenharia Mecânica) - Escola de Engenharia de São Carlos, da Universidade de São Paulo, São Paulo, 2005.

3 Coppini NL, Baptista EA, Souza E. Implantação de Sistema de Gerenciamento de Ferramentas de Usinagem - um estudo de caso. In: XXIX Encontro Nacional de Engenharia de Produção - ENEGEP. Salvador - BA, 2009.

4 Contador JC. Produtividade Fabril III: método para rápido aumento da produtividade fabril: redução de tempos inativos e do tempo de espera do material em processo. Gestão e Produção (UFSCar), São Carlos - SP, 1995; 2(2): 134-151.

5 Machado A. Comando Numérico Aplicado a Máquinas - Ferramenta. Ed. Ícone, São Paulo, 1990.

6 Ferraz JF. Arquitetura para Monitoramento e Supervisão Integrados de Processos de Usinagem em Maquinas com Controle Numérico Aberto. 2007. 163 f. Tese (Doutorado em Engenharia Mecânica) - Escola de Engenharia de São Carlos, da Universidade de São Paulo, São Paulo, 2007.

7 Pozo H. Administração de Recursos Materiais e Patrimoniais. São Paulo: Atlas, 2002.

8 Arbache FS, Santos AG, Montenegro C, Salles WF. Gestão de Logística, Distribuição e Trade Marketing. Editora FGV: Rio de Janeiro, 2004.

9 Chopra S, Meindl P. Gerenciamento da Cadeia de Suprimentos: estratégia, planejamento e operação. São Paulo: Prentice Hall, 2003.

10 Banzato E. WMS Warehouse Management System: sistema de gerenciamento de armazéns. São Paulo: IMAN,1998.

11 Dias JCQ. Logística Global e Macrologística. Lisboa: Edições Sílabo. 2005.

12 Casadevante Y Mújica, J.L.F. A Armazenagem na Prática. Lisboa: Editorial Pórtico, 1974.

13 Castiglioni JAM. "Logística Operacional: guia prático”. São Paulo: Érica, 2010.

14 Tomelero RL, Ferreira JCE. Benchmarking Enxuto para o Diagnóstico de Práticas e Performances de Empresas com Relação ao Gerenciamento de Ferramentas Incluindo Aspectos Ambientais. Anais do $7^{\circ}$ Congresso Brasileiro de Engenharia de Fabricação (COBEF). Rio de Janeiro, 2013.

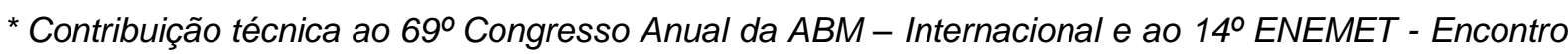
Nacional de Estudantes de Engenharia Metalúrgica, de Materiais e de Minas, 21 a 25 de julho de 2014, São Paulo, SP, Brasil.
} 